\title{
Crossfire DoS Attack and its Defence Mechanism
}

\author{
Sumer Shende \\ Department of Computer Science \\ Rajiv Gandhi Institute of Technology \\ Mumbai, India
}

\begin{abstract}
The Denial of Service (DoS) attacks represent a noteworthy danger to Internet users and administrations. Network threats are growing throughout the years, new sorts of DoS threats develop. One of such DoS attack is the Crossfire attack which is of extreme threat. In Crossfire, bots directs low intensity flows to a large number of servers. This paper presents a possible solution to this attack.
\end{abstract}

\section{General Terms}

Network Security Attack, Threats in Networks, Security Mechanisms.

\section{Keywords}

Crossfire attack, denial of service attack, network flooding.

\section{INTRODUCTION}

The point of the Crossfire attack is to block authorized clients from getting to a certain topographical region of the Internet, the Target Area. This range provides essential services to users.

The idea of the assault depends on the way that particular system connects, the Target Links, lead to both the Decoy Servers and the Target Area. Along these lines, an aggressor can utilize bots to surge the Target Links by sending movement just to the Decoy Servers. As a result, when the Target Links are overflowed, the Target Area gets to be inaccessible from whatever is left of the Internet. The proposed casualty doesn't know about the assault subsequent to there is not any attack activity bound to the Target Area.

This prevent legitimate traffic from flowing into a specific geographic region of the Internet, for which the attacker needs to surge a couple system joins in and around that area. We start by characterizing the two most regular terms utilized as a part of this paper: the target area and target link. At that point, we depict how an enemy outlines an assault utilizing the bots she controls.

To dispatch a Crossfire assault against an objective region, an attacker chooses an arrangement of open servers inside of the objective region and an arrangement of imitation servers encompassing the objective zone. These servers can be effectively found since they are browsed freely available servers. The arrangement of open servers is utilized to build an assault topology focused at the objective region, and the arrangement of distraction servers is utilized to make assault streams. At that point, the foe builds a "connection map", in particular the guide of layer-3 joins from her bot locations to those of the general population servers. (The contrasts between a connection map and a commonplace switch topology guide are talked about beneath.) Once the connection guide is made, the foe utilizes it to choose the best target interfaces whose flooding will adequately remove the objective region from the Internet. Next, the foe facilitates the bot-fake (server) streams to surge the objective connections, which would inevitably square the vast majority of the streams bound to the objective territory. This can be effortlessly done since target connections are shared by streams to the fake servers and target region. At last, the enemy chooses different disjoint arrangements of target connections for the same target range and surges them one set at once, in progression, to abstain from activating bot-server course changes. The three primary steps expected to dispatch the Crossfire assault comprise of the connection map development, assault setup, and bot coordination, as appeared in Fig. 1. Note that, to augment the length of time of the assault, the last step, to be specific the bot coordination step, is executed over and again by powerfully changing the arrangements of target connections. We portray each step of attack beneath [3]:-

\subsection{Link Map Creation}

The initial step of the Crossfire assault is the connection map development. The aggressor constructs a guide of the system joins along the ways from her bots to both the fake servers and people in general servers in the objective region utilizing traceroutes and handling their outcomes. Some ISPs often load-balance the traffic passing through their network, resulting in different routes between the same pairs of nodes Every bot executes various traceroutes to a destination to figure out if the same connections are crossed every time or not. Some ISPs regularly load-adjust the movement going through their system, bringing about diverse courses between the same sets of hubs. If so in the information accumulated by the traceroutes, the relating system connections are not considered as applicant target connections to be overwhelmed by the aggressor (because of the certain connection "security" through burden adjusting).

\subsection{Attack Setup}

After the link map has been built, the adversary utilizes the connections of the steady routes of the connection guide to decide the objective connections. The competitor target connections are sorted in view of the biggest number of courses and streams going through them, the stream thickness, and prompting the objective zone. On the off chance that a sure connection is utilized by countless, then its flooding can adequately disturb the entrance to the objective range. The assailant chooses numerous disjoint gatherings of potential target connections and surges one every time. The component of powerfully changing the arrangement of connections to surge improves the imperceptibility of the assault. The last target is to simultaneously surge every one of the connections of a sure arrangement of target connections every time keeping in mind the end goal to completely disturb target territory access for real activity. 


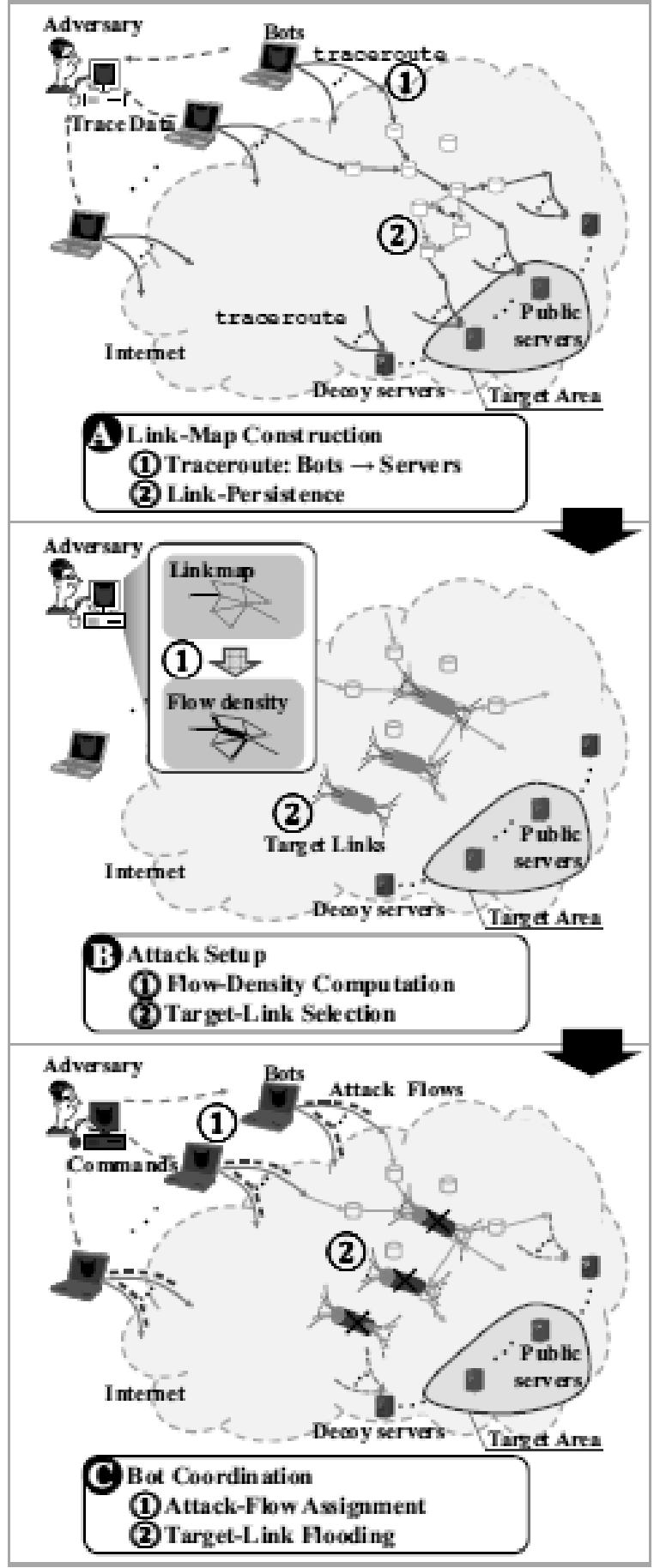

Fig 1: Steps of Crossover attack [3]

\subsection{Bot Coordination}

In the last a portion of the assault, the assailant appoints to her bots both the decoy servers to send activity to and their comparing stream rates and after that the bots begin the flooding. The task is made in a way that every stream from a bot to a fake server has low transmission capacity request while the chose target connections are overwhelmed by the total stream rate which surpasses the objective connection data transfer capacity. The streams created by the bots have low-rate so that no present security arrangement can order the activity as noxious. Moreover, the activity expected to surge the chose target connections is uniformly dispersed to various bots and imitation servers. In this way, the servers in the objective range are not able to distinguish the assault as no assault activity is bound to the objective region. The bait servers are additionally not able to distinguish the assault subsequent to the assault activity is appropriated in a substantial number of fake servers and there is not a sufficiently high data transfer capacity increment in every server to trigger an alert. After the task has completed, the bots begin producing the assault movement. The enemy can over and over execute the bot coordination piece of the assault by changing the arrangement of the objective connections keeping in mind the end goal to drag out its span and "misdirect" the protector.

\section{ASSUMPTIONS}

To assess the proposed solution to crossfire attack, we need to make a few assumptions on how the attacker responds to particular cases.

The aggressor begins with setting up and dispatching the assault taking after the same strides as in the Crossfire assault. Hence, the assailant builds a connection map for a sure target region, sets up the assault discovering the objective connections taking into account their stream thickness and after that appoints to her bots the imitations servers to send activity to. The aggressor proceeds with continually checking the beforehand built connection guide and the comparing stream thickness until any progressions are recognized. The aggressor can then figure out whether any rerouting along the ways between her bots and the distraction or target servers has happened. If so, the aggressor sets up again and launches the assault. The assailant has an altered assault spending plan and ought to apportion her assets as productively as could be allowed. The assault spending plan is characterized as the aggregate number of bots the foe utilizes to dispatch the assault and for our situation is thought to be settled.

There are numerous methodologies of fake server task to bots. A technique is chosen considering that the assault activity ought to be uniformly disseminated between the bots and the fake servers. Along these lines, the bots ought not to produce an excess of activity as they may be viewed as suspicious by the guard and every imitation server ought not to get a lot of movement so as an assault alert is not activated. Along these lines, the safeguard methodology gets to be troublesome, particularly as far as dependable recognition of the bots.

\section{DEFENCE MECHANISM}

Keeping in mind the end goal to recognize and mitigate the Crossfire assault, the issue is partitioned in two sections: the local approach that is empowered when the assault happens in the local domain and the inter-domain approach which is executed when the local approach is not able to handle the issue all alone or when the assault happens on a inter-domain level. The inter-domain methodology upgrades the identification and relief ability of the local one. We think about that as a Crossfire assault may emerge either inside of the nearby, endeavor, system or on the peering connections between the neighborhood space and its ISPs. In the extent of this proposal, we expect that a Crossfire assault can't happen inside of an ISP system because of the wealth of its assets. We additionally consider that assault movement does not start from the nearby, endeavor system.

In Figure 2, our detection approach in a local domain area is exhibited. Each connection in the nearby system is always checked. If there should arise an occurrence of a serious connection blockage, we check if another has happened previously (i.e., if our security calculation has in any event 
been executed once). This is on the grounds that we might want to correspond a present blockage with an old one. If this is run for the first time, then we check if a rerouting is possible to sidestep the congested connection. If the local topology permits an optional route, then a selection of the congested streams is rerouted and the relating sources are recorded.

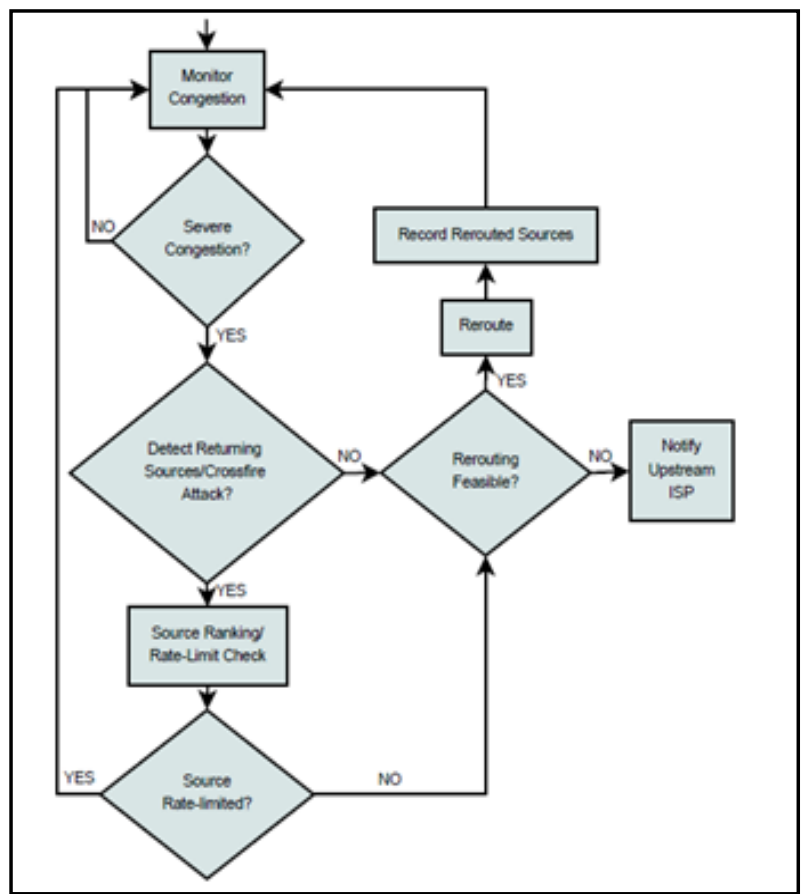

Fig 2: Crossfire Detection and mitigation model [5]

We utilize destination-based rerouting for the congested streams subsequent to the quantity of the destinations of the congested movement is far not exactly the quantity of the congested streams. We need to recall that in the Crossfire assault a connection is overflowed by an expansive number of low-rate movement streams. Our rerouting technique leads a portion of the congested movement in courses disjoint to the beforehand ascertained by the assailant target connections and possibly disjoint to the new ones (the computed ones after the attacker has distinguished and responded to the route shifts). The outline objective of the rerouting is to "constrain" a potential attacker to persevere in flooding target connections utilizing the same sources yet as a part of movement bound to diverse destinations (fake servers). The clog is alleviated and vindictive movement can be recognized if the same sources hold on in further blockage occasions. The current and the past flooding occasions are related, and if the aforementioned craved response of the potential aggressor is recognized, then the comparing sources are stamped as suspicious, increasing a comparing counter. This activity building instrument upholds relief of the congested movement and may prompt the identification of the vindictive activity.

This rerouting may build the expense of the assault as a few bots may be required to send more streams to the same fake servers to surge the objective connection on the off chance that no more imitation servers become possibly the most important factor as in this case. Our methodology endeavors to build the likelihood of identifying the assault at the fake servers by verifiably constraining the aggressor to allot more streams to certain decoy servers (more transmission capacity is gotten by the decoy servers).
The objective connection is overwhelmed and the wellsprings of the streams that were beforehand rerouted are available in the congested connection. Since the rerouted sources quit sending movement towards the rerouting ways and are currently present in the overflowed join, we can accept that these are suspicious sources. Therefore, these sources are stamped in our endeavor to distinguish the vindictive activity. For whatever length of time that the assault is persevering and our topology permits various rerouting, the malicious movement can be recognized with higher certainty.

The congested movement is rerouted as before the length of the topology permits it. In the event that there are not any further option courses for the congested streams or the topology does not permit any optional ways, then the comparing upstream ISP ought to be told to expand our identification and alleviation endeavors. The controller of the neighborhood system corresponds with the one of the supplier system to reroute the congested courses through another peering connection of this supplier or of a teaming up one and advises the nearby system about the rerouting condition of the reported streams. The neighborhood calculation proceeds with its execution as some time recently. The benefit of our methodology is that both the neighborhood system and the working together suppliers are permitted to not uncover data about their systems.

\section{CONCLUSION}

In this paper we have reviewed Crossfire attack with distribute low intensity packet flooding. Planning, assessing an assault countermeasure to a responsive Crossfire assault requires a ton of exertion. We have also studied a possible solution to detect and mitigate this attack in real time. In spite of a few weaknesses, this system can form the basis for preventing such a DoS attack.

\section{ACKNOWLEDGMENTS}

During the course of this work, the Computer Department of Rajiv Gandhi Institute of Technology with its faculty members have been most cooperative. Their help and council helped me in the completion of this composition. I am grateful to Prof. Gautam Borkar for his insightful suggestions and for guiding me throughout my study.

\section{REFERENCES}

[1] Defense and Monitoring Model for Distributed Denial of Service Attacks. Article Procedia Computer Science, Volume 10, 2012, Pages 1052-1056

[2] Poseidon: Mitigating Interest Flooding DDoSAttacks in Named Data Networking. 978-1-4799-0537-9/13 IEEE pg 630-638 @2013

[3] The Crossfire Attack, 2013 IEEE Symposium on Security and Privacy. 1081-6011/13 \$26.00 (C) 2013 IEEE. DOI 10.1109/SP.2013.19

[4] A distributed detecting method for SYN flood attacks and its implementation using mobile agents MATES'09 Proceedings of the 7th German conference on Multiagent system technologies Pages 91-102.

[5] Cross-domain DoS link-flooding attack detection and mitigation using SDN principles. Master Thesis MA2013-18

[6] DDoS Attack Detection Using Fast Entropy Approach on Flow- Based Network TrafficOriginal Research Article. Procedia Computer Science, Volume 50, 2015, Pages 3036. 